\title{
Personnel Selection Method Based on Personnel-Job Matching
}

\author{
Li Wang, Xilin Hou (Corresponding author) \& Lili Zhang \\ School of Business Administration, University of Science and Technology Liaoning \\ 185 Qianshan Zhong Road, Anshan 114051, Liaoning, China \\ E-mail: hanxinxiwang@126.com, wangli0906@yeah.net, zhangliqinzhi@126.com
}

\author{
Received: November 5, 2012 \\ Accepted: November 26, $2012 \quad$ Online Published: January 5, 2013 \\ doi:10.5430/ijba.v4n1p105 \\ URL: http://dx.doi.org/10.5430/ijba.v4n1p105
}

The research is financed by China Postdoctoral. No.2012M511153; Liaoning Provincial. No.W2012045; School youth fund. No. 2011zx09; City Social Sciences. No. as20111005.

\begin{abstract}
The existing personnel selection decisions in practice are based on the evaluation of job seeker's human capital, and it may be difficult to make personnel-job matching and make each party satisfy. Therefore, this paper puts forward a new personnel selection method by consideration of bilateral matching. Starting from the employment thoughts of "satisfy", the satisfaction evaluation indicator system of each party are constructed. The multi-objective optimization model is given according to the job seekers satisfaction and jobs satisfaction. By the method of fuzzy multi-objective optimization model, based on the thought of "disadvantage" is as shorter as better, the selection results are obtained. The effectiveness and feasibility of the proposed method is illustrated by an example.
\end{abstract}

Keywords: Personnel selection, Matching, Fuzzy multi-objective optimization model

\section{Introduction}

With the development of society, human capital is becoming more and more important. Personnel selection is the beginning part of the whole human resources management, how to select the fit personnel is a hot top in the field of business management. Personnel-job matching has the important influence on improving company performance and gaining competitive advantage (Golec, A. \& Kahya, E., 2007). In fact, most of the companies select personnel from the single perspective of the organization, they ignore the needs of personnel, it is very dangerous, because it may cause a series of bad results, such as dissatisfaction, leaving, and so on. Based on the above questions of unilateral thought, this paper puts forward a new angle of view, in the selection process, we take consideration of both the satisfaction of personnel and job, and in order to reduce the risk of employment, we make the lowest point of competency and need as much as possible as objective function, so we can get the double win results.

\section{Satisfaction Indication System Construction}

\subsection{Job Seeker Satisfaction Indication System}

According to the references and field research, and combine with the experts' opinions, competency model of job satisfaction is shown in Figure 1.

\subsection{Job Satisfaction Indication System}

According to the references and field research, and combine with the experts' opinions, the needs job seekers with their work are shown in Figure 2.

\section{Selection Method Based on Personnel-Job Bilateral Matching}

\subsection{Personnel-Job Bilateral Matching Problem Description}

Assuming that job seekers is $\mathrm{X}=\left\{\mathrm{X}_{1}, \mathrm{X}_{2}, \ldots, \mathrm{X}_{\mathrm{n}}\right\}$, where $\mathrm{X}_{\mathrm{i}}$ expresses the $\mathrm{i}$-th job seeker; The set of jobs is $\mathrm{Y}=$ $\left\{Y_{1}, Y_{2}, \ldots Y_{m}\right\}$, where $Y_{j}$ represents the $j$-th job. Evaluation index set of matching satisfaction given by jobs to job seekers is $B=\left(B_{1}, B_{2}, \ldots, B_{f}\right)$, where $B_{h}$ says the $h$-th index $(h=1,2 \ldots f)$; the weight vector of evaluation index corresponding to the $\mathrm{B}$ is $\mathrm{w}=\left(\mathrm{w}_{1}, \mathrm{w}_{2}, \ldots \mathrm{w}_{\mathrm{f}}\right)$, where $\mathrm{w}_{\mathrm{h}}$ represents the index weight of $\mathrm{B}_{\mathrm{h}}, 0 \leq \mathrm{w}_{\mathrm{h}} \leq 1, \sum_{h}=1$; multi-criteria linguistic assessment information of matching satisfaction degree given by jobs to job seekers is 
recorded by $\bar{A}_{h}=\left[\bar{a}_{h i j}\right]_{n \times m}, \quad \bar{a}_{h i j}$ is expressed as $\mathrm{Y}_{\mathrm{j}}$ given for $\mathrm{X}_{\mathrm{i}}$ on index $\mathrm{B}_{\mathrm{h}}$ 's matching satisfaction language evaluation value. Assuming that evaluation index set of matching satisfaction given by job seekers to jobs is $\mathrm{D}=$ $\left(D_{1}, D_{2}, \ldots . D_{k}\right)$, where $D_{q}$ says the $q$-th index $(q=1,2, \ldots k)$; the weight vector of evaluation index corresponding to the $\mathrm{D}$ is $\mathrm{v}=\left(\mathrm{v}_{1}, \mathrm{v}_{2}, \ldots \mathrm{v}_{\mathrm{k}}\right)$, where $\mathrm{v}_{\mathrm{q}}$ represents the index weight of $\mathrm{D}_{\mathrm{q}}, 0 \leq \mathrm{V}_{\mathrm{q}} \leq 1, \sum^{k} v_{q}=1$; multi-criteria linguistic assessment information of matching satisfaction degree given by job ${ }^{q=1}$ seekers to jobs is recorded by $\bar{B}_{q}=\left[\bar{b}_{q i j}\right]_{n \times n}$, where $\bar{b}_{q i j}$ is expressed as $\mathrm{X}_{\mathrm{i}}$ given for $\mathrm{Y}_{\mathrm{j}}$ on index $\mathrm{D}_{\mathrm{q}}$ 's matching satisfaction language evaluation value. We can get $\mathrm{W}$ and $\mathrm{v}$ through the simple average method criterion. Assuming that seekers or jobs select an element from predefined language evaluation set $\mathrm{S}$ as his preference evaluation, that is to say, provide $\bar{a}_{h i j}$ and $\bar{b}_{q i j} . \mathrm{S}$ is constituted by the ordered collection of an odd number of elements, $\mathrm{S}=\left\{\mathrm{S}_{1}, \mathrm{~S}_{2}, \ldots \mathrm{S}_{\mathrm{T}}\right\}, \mathrm{S}_{\mathrm{i}}$ is the i-th language phrase, $\mathrm{i}=\{0,1, \ldots \mathrm{T}\}$, According to the actual situation, Language phrase set consists of five or seven granularity language phrase by predefined.

In summary, the problem to be solved in this paper is job seekers and jobs on linguistic assessment information of multiple Indicator satisfaction $\left(\bar{A}_{h}\right.$ and $\bar{B}_{q}$ ) and the weight vector of evaluation index (w and v), through some sort of decision analysis methods, let job seekers and jobs match reasonably, trying to make both the needs or requirements achieve satisfactory results.

\subsection{Fuzzy Multi-Objective Decision Method}

In order to deal with linguistic assessment information, take language phrase into triangular fuzzy number (Li, R.J., 1999). It's recorded in formula (1).

$$
\tilde{A}=\left(\mathrm{d}^{1}, \mathrm{~d}^{s}, \mathrm{~d}^{\mathrm{r}}\right)=[\max \{(\mathrm{i}-1) / \mathrm{T}, 0\}, \mathrm{i} / \mathrm{T}, \min \{(\mathrm{i}+1) / \mathrm{T}, 1\}], \mathrm{i}=(0,1,2, \ldots \mathrm{T})
$$

Transform evaluation matrix $\bar{A}_{h}=\left[\bar{a}_{h i j}\right]_{n \times m}$ and $\bar{B}_{q}=\left[\bar{b}_{q i j}\right]_{n \times m}$ into the form of triangular fuzzy numbers $\tilde{A}_{h}=\left[\tilde{a}_{h i j}\right]_{n \times m}, \tilde{B}_{q}=\left[\tilde{b}_{q i j}\right]_{n \times m}$, fuzzy comprehensive evaluation value of matching satisfaction degree given by jobs to job seekers is recorded as $\tilde{\alpha}_{i j}=\left(\alpha_{i j}^{l}, \alpha_{i j}^{s}, \alpha_{i j}^{r}\right)$, and fuzzy comprehensive evaluation value of matching satisfaction degree given by job seekers to jobs is recorded as $\tilde{\beta}_{i j}=\left(\beta_{i j}^{l}, \beta_{i j}^{s}, \beta_{i j}^{r}\right)$. The calculation formula of $\tilde{\alpha}_{i j}$ and $\tilde{\beta}_{i j}$ are respectively expressed in formula (2).

$$
\begin{aligned}
& \tilde{\alpha}_{i j}=\left(\alpha_{i j}^{l}, \alpha_{i j}^{s}, \alpha_{i j}^{r}\right)=\left[\sum_{h=1}^{f} w_{h} \alpha_{h i j}^{l}, \sum_{h=1}^{f} w_{h} \alpha_{h i j}^{s}, \sum_{h=1}^{f} w_{h} \alpha_{h i j}^{r}\right], \mathrm{i}=1,2, \ldots \mathrm{n} ; \mathrm{j}=1,2, \ldots \mathrm{m} \\
& \tilde{\beta}_{i j}=\left(\beta_{i j}^{l}, \beta_{i j}^{s}, \beta_{i j}^{r}\right)=\left[\sum_{q=1}^{k} v_{q} b_{q i j}^{l}, \sum_{q=1}^{k} v_{q} b_{q i j}^{s}, \sum_{q=1}^{k} v_{q} b_{q i j}^{r}\right], \mathrm{i}=1,2, \ldots \mathrm{n} ; \mathrm{j}=1,2, \ldots \mathrm{m}
\end{aligned}
$$

Assuming that $x_{i j}$ means the matching of $\mathrm{X}_{\mathrm{i}}$ and $\mathrm{Y}_{\mathrm{j}}$. According to Xi Chen \& Zhiping Fan (2009), the fuzzy multi-objective optimization model is shown in formula (3).

$$
\begin{aligned}
& M a x z_{1}=\sum_{i=1}^{n} \sum_{j=1}^{m} \tilde{\alpha}_{i j} x_{i j} \\
& M a x z_{2}=\sum_{i=1}^{n} \sum_{j=1}^{m} \tilde{\beta}_{i j} x_{i j} \\
& \text { s.t. } \\
& \sum_{j=1}^{m} x_{i j} \leq 1 \quad i=1,2, \ldots n \\
& \sum_{i=1}^{n} x_{i j} \leq q_{j} \quad j=1,2, \ldots m \\
& x_{i j}=0 \text { or } 1
\end{aligned}
$$

For the above formula (3), there are two objective functions, which are expressed respectively as: make jobs to job seekers satisfaction maximum and make job seekers to jobs satisfaction maximum as much as possible; there are three constraint conditions, which are expressed respectively as: $\mathrm{X}_{\mathrm{i}}$ is assigned to one post at most; $\mathrm{Y}_{\mathrm{j}}$ can recruit $q_{j}$ employees at most; $x_{i j}=0$ says $\mathrm{Xi}$ and $\mathrm{Yj}$ don't match, $x_{i j}=1$ says $\mathrm{Xi}$ and $\mathrm{Yj}$ is matching.

Here the method given in Zimmermann, H.J (1978) \& Lai, Y. \& Hwang, C. (1992), in order to solve the above optimization model and considering the objective function with fuzzy number case, transform the model (3) into the optimization model which includes the form of definition digital. It's shown in formula (4). 


$$
\begin{aligned}
& z_{1}^{l}=\sum_{i=1}^{n} \sum_{j=1}^{m} \alpha_{i j}^{l} x_{i j}, z_{1}^{s}=\sum_{i=1}^{n} \sum_{j=1}^{m} \alpha_{i j}^{s} x_{i j}, z_{1}^{r}=\sum_{i=1}^{n} \sum_{j=1}^{m} \alpha_{i j}^{r} x_{i j} \\
& z_{2}^{l}=\sum_{i=1}^{n} \sum_{j=1}^{m} \beta_{i j}^{l} x_{i j}, z_{2}^{s}=\sum_{i=1}^{n} \sum_{j=1}^{m} \beta_{i j}^{s} x_{i j}, z_{2}^{r}=\sum_{i=1}^{n} \sum_{j=1}^{m} \beta_{i j}^{r} x_{i j}
\end{aligned}
$$

In line with the human-oriented idea, this paper takes consideration of risk, tries to make the risk lowest by maximizing $z_{1}^{l}, z_{2}^{l}, z_{1}^{s}-z_{1}^{l}, z_{1}^{r}-z_{1}^{l}, z_{2}^{s}-z_{2}^{l}, z_{2}^{r}-z_{2}^{l}$, transform triangular fuzzy number of objective function into clear number, so fuzzy multi-objective optimization model (3) can be transformed into following linear multi-objective programming model. It's shown in formula (5).

$$
\begin{aligned}
& M a x z_{1}^{\prime}=z_{1}^{l} \\
& \text { Maxz } z_{1}^{\prime \prime}=\left(z_{1}^{s}-z_{1}^{l}\right)=\sum_{i=1}^{n} \sum_{j=1}^{m}\left(\alpha_{i j}^{s}-\alpha_{i j}^{l}\right) x_{i j} \\
& M a x z_{1}^{\prime \prime \prime}=\left(z_{1}^{r}-z_{1}^{l}\right)=\sum_{i=1}^{n} \sum_{j=1}^{m}\left(\alpha_{i j}^{r}-\alpha_{i j}^{l}\right) x_{i j} \\
& M a x z_{2}^{\prime}=z_{2}^{l} \\
& M a x z_{2}^{\prime \prime}=\left(z_{2}^{s}-z_{2}^{l}\right)=\sum_{i=1}^{n} \sum_{j=1}^{m}\left(\beta_{i j}^{s}-\beta_{i j}^{l}\right) x_{i j} \\
& \text { Maxz } z_{2}^{\prime \prime \prime}=\left(z_{2}^{r}-z_{2}{ }^{l}\right)=\sum_{i=1}^{n} \sum_{j=1}^{m}\left(\beta_{i j}^{r}-\beta_{i j}^{l}\right) x_{i j} \\
& \text { s.t. } \\
& \sum_{j=1}^{m} x_{i j} \leq 1 \quad i=1,2, \ldots n \\
& \sum_{i=1}^{n} x_{i j} \leq q_{j} \quad j=1,2, \ldots m \\
& x_{i j}=0 \text { or } 1 \quad i=1,2, \ldots n ; j=1,2, \ldots m
\end{aligned}
$$

Respectively set and obtain positive ideal solution and negative ideal solution of each of the above objective function, it's shown in formula (6).

$$
\begin{aligned}
& z_{1}^{\prime+}=\operatorname{Max} z_{1}^{l}, z_{1}^{\prime-}=\operatorname{Min} z_{1}^{l} \\
& z_{1}^{\prime \prime+}=\operatorname{Max}\left(z_{1}^{s}-z_{1}^{l}\right), z_{1}^{\prime \prime-}=\operatorname{Min}\left(z_{1}^{s}-z_{1}^{l}\right) \\
& z_{1}^{\prime \prime \prime}=\operatorname{Max}\left(z_{1}^{r}-z_{1}^{l}\right), z_{1}^{\prime \prime \prime}=\operatorname{Min}\left(z_{1}^{r}-z_{1}^{l}\right) \\
& z_{2}^{\prime+}=\operatorname{Max} z_{2}^{l}, z_{2}^{\prime-}=\operatorname{Min} z_{2}^{l} \\
& z_{2}^{\prime \prime+}=\operatorname{Max}\left(z_{2}^{s}-z_{2}^{l}\right), z_{2}^{\prime \prime-}=\operatorname{Min}\left(z_{2}^{s}-z_{2}^{l}\right) \\
& z_{2}^{\prime \prime \prime+}=\operatorname{Max}\left(z_{2}^{r}-z_{2}^{l}\right), z_{2}^{\prime \prime \prime}=\operatorname{Min}\left(z_{2}^{r}-z_{2}^{l}\right)
\end{aligned}
$$

According to the positive and negative ideal solution of each objective function, we can calculate the corresponding membership function $\mu\left(z_{1}^{\prime}\right), \mu\left(z_{1}^{\prime \prime}\right), \mu\left(z_{1}^{\prime \prime \prime}\right)$ and $\mu\left(z_{2}^{\prime}\right), \mu\left(z_{2}^{\prime \prime}\right), \mu\left(z_{2}^{\prime \prime \prime}\right)$, its computation formula respectively are recorded in formula (7). 


$$
\begin{aligned}
& \mu\left(z_{1}^{\prime}\right)=\left\{\begin{array}{cc}
1, & z_{1}^{\prime} \succ z_{1}^{\prime+} \\
\frac{z_{1}^{\prime}-z_{1}^{\prime}}{z_{1}^{\prime+}-z_{1}^{\prime-}}, & z_{1}^{\prime-} \leq z_{1}^{\prime} \leq z_{1}^{\prime+} \\
0 & z_{1}^{\prime} \prec z_{1}^{\prime-}
\end{array}\right. \\
& \mu\left(z_{1}^{\prime \prime}\right)=\left\{\begin{array}{cc}
1, & z_{1}^{\prime \prime} \succ z_{1}^{\prime \prime+} \\
\frac{z_{1}^{\prime \prime}-z_{1}^{\prime \prime}-}{z_{1}^{\prime \prime+}-z_{1}^{\prime \prime-}}, & z_{1}^{\prime \prime-} \leq z_{1}^{\prime \prime} \leq z_{1}^{\prime \prime+} \\
0 & z_{1}^{\prime \prime} \prec z_{1}^{\prime \prime-}
\end{array}\right. \\
& \mu\left(z_{1}^{\prime \prime \prime}\right)=\left\{\begin{array}{cc}
1, & z_{1}^{\prime \prime \prime} \succ z_{1}^{\prime \prime \prime+} \\
\frac{z_{1}^{\prime \prime \prime}-z_{1}^{\prime \prime \prime}-}{z_{1}^{\prime \prime \prime}-z_{1}^{\prime \prime \prime}}, & z_{1}^{\prime \prime \prime} \leq z_{1}^{\prime \prime \prime} \leq z_{1}^{\prime \prime \prime+} \\
0 & z_{1}^{\prime \prime \prime} \prec z_{1}^{\prime \prime \prime}-
\end{array}\right. \\
& \mu\left(z_{2}^{\prime}\right)=\left\{\begin{array}{crl}
1, & z_{2}^{\prime} \succ z_{2}^{\prime+} \\
\frac{z_{2}^{\prime}-z_{2}^{\prime-}}{z_{2}^{\prime+}-z_{2}^{\prime-}}, & z_{2}^{\prime-} \leq z_{2}^{\prime} \leq z_{2}^{\prime+} \\
0 & z_{2}^{\prime} \prec z_{2}^{\prime-}
\end{array}\right. \\
& \mu\left(z_{2}^{\prime \prime}\right)=\left\{\begin{array}{crl}
1, & z_{2}^{\prime \prime} \succ z_{2}^{\prime \prime+} \\
\frac{z_{2}^{\prime \prime}-z_{2}^{\prime \prime}-}{z_{2}^{\prime \prime}-z_{2}^{\prime \prime}-}, & z_{2}^{\prime \prime-} \leq z_{2}^{\prime \prime} \leq z_{2}^{\prime \prime+} \\
0 & z_{2}^{\prime \prime} \prec z_{2}^{\prime \prime-}
\end{array}\right. \\
& \mu\left(z_{2}^{\prime \prime \prime}\right)=\left\{\begin{array}{cc}
1, & z_{2}^{\prime \prime \prime} \succ z_{2}^{\prime \prime \prime+} \\
\frac{z_{2}^{\prime \prime \prime}-z_{2}^{\prime \prime \prime}-}{z_{2}^{\prime \prime \prime}-z_{2}^{\prime \prime \prime}-}, & z_{2}^{\prime \prime \prime} \leq z_{2}^{\prime \prime \prime} \leq z_{2}^{\prime \prime \prime+} \\
0 & z_{2}^{\prime \prime \prime} \prec z_{2}^{\prime \prime \prime}-
\end{array}\right.
\end{aligned}
$$

According to the calculated membership function, through Zimmermann, H.J (1978), transform formula (5) which expresses multi-objective programming model into the following single-objective linear programming model. It's shown in formula (8).

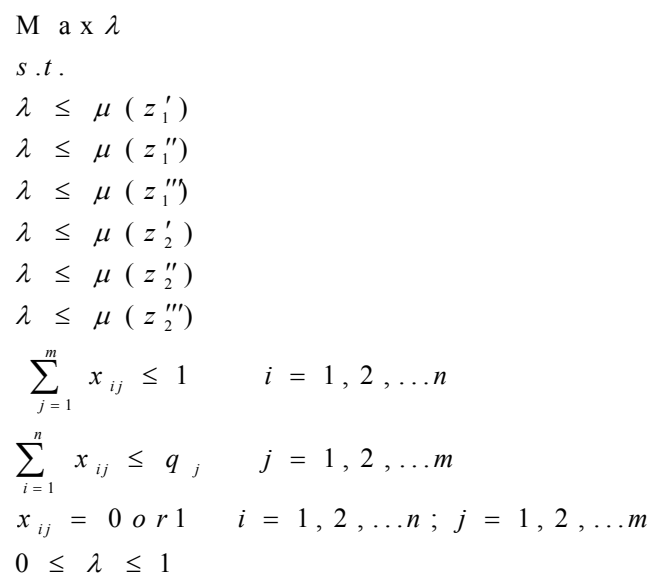

In order to solve the above single-objective linear programming model, we can use Lingo software, or programming way.

\section{Numerical Example}

An iron and steel enterprise in China intends to select knowledge workers in four positions $\left(\mathrm{Y}_{1}, \mathrm{Y}_{2}, \mathrm{Y}_{3}, \mathrm{Y}_{4}\right)$, after qualifying examination, eight job seekers $\left(\mathrm{X}_{1}, \mathrm{X}_{2}, \mathrm{X}_{3}, \mathrm{X}_{4}, \mathrm{X}_{5}, \mathrm{X}_{6}, \mathrm{X}_{7}, \mathrm{X}_{8}\right)$ come to apply for the job. According to fuzzy multi-objective decision method above, use seven granularity language evaluation set as shown in table 1 in order to make the job seekers and jobs achieve satisfactory at the same time. Through the statistical analysis, using the simple average criterion, draw the corresponding weight vector: $\mathrm{w}=\left(\mathrm{w}_{1}, \mathrm{w}_{2}, \mathrm{w}_{3}, \mathrm{w}_{4}, \mathrm{w}_{5}\right)=[0.200,0.200,0.200$, $0.200,0.200]$. At the same time, through the statistical analysis, draw the corresponding weight vector using the simple average criterion: $\mathrm{v}=\left(\mathrm{v} 1, \mathrm{v}_{2}, \mathrm{v}_{3}, \mathrm{v}_{4}, \mathrm{v}_{5}\right)=[0.200,0.200,0.200,0.200,0.200]$.

Table 2 shows multi-criteria linguistic assessment information of matching satisfaction degree given by jobs to job 
seekers, the information for job seekers is given through comparative analysis of actual level and reference post competency required level in the index. That is: relative to the job in the index requirements concerned, the job seekers' competent degree. Table 3 shows multi-criteria linguistic assessment information of matching satisfaction degree given by job seekers to jobs, this information reflects the satisfaction degree of job seekers on the job indicators. Table 2 and table 3 shows that multi-index matching satisfaction evaluation information is the form of linguistic assessment information. Assuming that every job invite to consider the number is $q_{j}=3(j=1,2,3,4)$. Based on the given method, first, transform the multi-index linguistic assessment information given by employees and positions into the form of triangular fuzzy numbers, then use formula (2) for multiple Indicator information assembled computing, respectively get: fuzzy comprehensive evaluation value of matching satisfaction degree given by jobs to job seekers and fuzzy comprehensive evaluation value of matching satisfaction degree given by job seekers to jobs. Respectively as shown in Table 4 and Table 5.

According to the formula (3), establish a fuzzy multi-objective optimization model about jobs and job seekers matching, then in accordance with the method given, transform it into single objective linear programming model.

We can use Lingo optimization package to solve the optimization model here, by calculating, we get six positive and negative ideal solution of objective function as shown in table 6 .

Thus obtain the optimal solution of the optimization model:

$\mathrm{x}_{11}=0 ; \mathrm{x}_{12}=0 ; \mathrm{x}_{13}=0 ; \mathrm{x}_{14}=1 ; \mathrm{x}_{21}=0 ; \mathrm{x}_{22}=0 ; \mathrm{x}_{23}=1 ; \mathrm{x}_{24}=0 ; \mathrm{x}_{31}=0 ; \mathrm{x}_{32}=1 ; \mathrm{x}_{33}=0 ; \mathrm{x}_{34}=0 ; \mathrm{x}_{41}=0 ; \mathrm{x}_{42}=0 ; \mathrm{x}_{43}=1 ; \mathrm{x}_{44}=0 ;$

$\mathrm{x}_{51}=0 ; \mathrm{x}_{52}=1 ; \mathrm{x}_{53}=0 ; \mathrm{x}_{54}=0 ; \mathrm{x}_{61}=1 ; \mathrm{x}_{62}=0 ; \mathrm{x}_{63}=0 ; \mathrm{x}_{64}=0 ; \mathrm{x}_{71}=0 ; \mathrm{x}_{72}=0 ; \mathrm{x}_{73}=0 ; \mathrm{x}_{74}=1 ; \mathrm{x}_{81}=1 ; \mathrm{x}_{82}=0 ; \mathrm{x}_{83}=0 ; \mathrm{x}_{84}=0$.

According to the optimal solution of the above-obtained, knowledge workers and job matching results is: $\mathrm{Y} 1$ matches with X6, X8; Y2 match with X3, X5; Y3 matches with X2,X4; Y4 matches with X1,X7.

\section{Conclusions and Innovations}

This paper puts forward a new personnel selection method according to the satisfaction of both job seekers and organizations which can obtain more effective personnel; it can be used as decision making reference. It has the following innovation points:

Firstly, it is different from the only recruiting parts with decision making power, the decision making methods takes consideration of job seeker' needs, it is human-oriented, under the guide of this, the selection result will be more sustainable.

Secondly, for the consideration of risk, this paper makes the risk lowest from the following two perspectives: on the one hand, from the perspective of recruiting organization, by the application of this method, it can get the least disadvantage about competency personnel on the whole. On the other hand, from the perspective of job seeks, by the application of this method, it can get the least dissatisfaction about the job from an overall point of view.

Last but not the least, this paper constructs a new optimization model for personnel selection, the way for changing the fuzzy to the precise number about the goal function is new, it can be seen as an innovation try, which can be used as reference for other fuzzy optimization methods.

\section{References}

Chen, Xi, \& Fan, Zhiping. (2009). Bilateral matching decision method research. Shen Yang: Business Administration College of northeastern university. [Online] Available: http://cdmd.cnki.com.cn/Article/CDMD-10145-1012300721.htm

Golec, A., \& Kahya, E. (2007). A fuzzy model for competency-based employee evaluation and selection. Computers \& Industrial Engineering, 52(1), 143-161. [Online] Available: http://www.sciencedirect.com/science/article/pii/S0360835206002026

Lai, Y., \& Hwang, C. (1992). A new approach to some possibilistic linear programming problems. Fuzzy Sets and Systems, 49(2), 121-133. [Online] Available: http://www.sciencedirect.com/science/article/pii/016501149290318X

Li, R. J. (1999). Fuzzy method in group decision making. Computers and Mathematics with Application, 38(1), 91-101. [Online] Available: http://www.sciencedirect.com/science/article/pii/S0898122199001728

Zimmermann, H. J. (1978). Fuzzy Programming and linear Programming with several objective functions. Fuzzy Sets and Systems, 1(1), 45-55. Available: http://www.sciencedirect.com/science/article/pii/0165011478900313 
Table 1. Linguistic terms with 7 granularity and corresponding triangular fuzzy numbers

\begin{tabular}{|l|c|}
\hline \multicolumn{1}{|c|}{ language phrases } & corresponding triangular fuzzy numbers \\
\hline $\begin{array}{l}\mathrm{S}_{0}=\mathrm{AP}(\text { extremely poor/extremely low/ extremely } \\
\text { dissatisfied) }\end{array}$ & $(0,0,0.167)$ \\
\hline $\mathrm{S}_{1}=\mathrm{VP}($ very poor / very low / very dissatisfied) & $(0,0.167,0.333)$ \\
\hline $\mathrm{S}_{2}=\mathrm{P}($ poor / low / dissatisfied) & $(0.167,0.333,0.5)$ \\
\hline $\mathrm{S} 3=\mathrm{M}(\mathrm{medium} / \mathrm{medium} /$ general) & $(0.333,0.5,0.667)$ \\
\hline $\mathrm{S} 4=\mathrm{G}$ (good/ high / satisfied ) & $(0.5,0.667,0.833)$ \\
\hline $\mathrm{S} 5=\mathrm{VG}($ very good /very high/very satisfied ) & $(0.667,0.833,1)$ \\
\hline $\begin{array}{l}\mathrm{S} 6=\mathrm{AG}(\text { extremely good / extremely high / extremely } \\
\text { satisfied) }\end{array}$ & $(0.833,1,1)$ \\
\hline
\end{tabular}

Table 2. Multi-criteria linguistic assessment information of matching satisfaction degree given by jobs to job seekers $\left(\bar{A}_{h}=\left[\bar{a}_{h i j}\right]_{n \times m}\right)$

\begin{tabular}{|c|c|c|c|c|c|c|c|c|c|c|c|c|c|c|}
\hline $\mathrm{B}_{1}$ & \multicolumn{3}{|c|}{$\mathrm{B}_{2}$} & \multicolumn{4}{|c|}{$\mathrm{B}_{3}$} & \multicolumn{3}{|c|}{$\mathrm{B}_{4}$} & \multicolumn{4}{|c|}{$\mathrm{B}_{5}$} \\
\hline $\begin{array}{llll}Y_{1} & Y_{2} & Y_{3} & Y_{4}\end{array}$ & $\mathrm{Y}_{1} \mathrm{Y}_{2}$ & $\mathrm{Y}_{3}$ & & & $\mathrm{Y}_{2}$ & $\mathrm{Y}_{3} \mathrm{Y}$ & & $\mathrm{Y}_{1} \mathrm{Y}_{2}$ & $\mathrm{Y}_{3}$ & & $Y_{1}$ & $\mathrm{Y}_{2} \mathrm{Y}$ & $Y_{3} \mathrm{Y}_{4}$ & \\
\hline VP VP VP VP & $\mathrm{M} \quad \mathrm{M}$ & $\mathrm{M}$ & & VP & VP & $\mathrm{VP}$ & & $\mathrm{P} \quad \mathrm{P}$ & VP & $\mathrm{P}$ & & $\mathrm{P}$ & $\mathrm{G}$ & \\
\hline M $M \quad M \quad M$ & $\mathrm{M} \quad \mathrm{P}$ & $\mathrm{P}$ & $\mathrm{M}$ & M & M & $\mathrm{M}$ & $\mathrm{M}$ & VG VG & VG & VG & $\mathrm{P}$ & M & M & M \\
\hline $\mathrm{M} \quad \mathrm{M} \quad \mathrm{P}$ & G & $\mathrm{P}$ & G & VG & VG & $\mathrm{P}$ & G & M AG & AG & VG & M & VG & AG & $\mathrm{P}$ \\
\hline $\begin{array}{llll}P & P & V P & P\end{array}$ & VG VG & VG & VG & & G & G & $\mathrm{P}$ & M VP & $\mathrm{M}$ & $\mathrm{P}$ & $\mathrm{P}$ & $\mathrm{P}$ & $\mathrm{G}$ & $\mathrm{P}$ \\
\hline VGVGVG VG & AG AG & AG & AG & G & G & G & G & M VG & AP & G & G & G & G & $\mathrm{P}$ \\
\hline M M M & M M & VG & $M$ & $\mathrm{P}$ & M & M & M & VP & M & G & AG & $\mathrm{AG}$ & $\mathrm{M}$ & VP \\
\hline $\mathrm{G} \quad \mathrm{M} \quad \mathrm{G}$ & M & $\mathrm{G}$ & VG & M & $\mathrm{P}$ & G & G & G & $\mathrm{M}$ & $\mathrm{M}$ & G & VG & $\mathrm{M}$ & $\mathrm{P}$ \\
\hline $\mathrm{M} P$ & VG & $\mathrm{P}$ & $\mathrm{P}$ & G & $\mathrm{M}$ & $\mathrm{M}$ & $\mathrm{P}$ & M VG & G & $\mathrm{P}$ & $\mathrm{M}$ & $\mathrm{G}$ & $\mathrm{G}$ & $\mathrm{M}$ \\
\hline
\end{tabular}

Table 3. Multi-criteria linguistic assessment information of matching satisfaction degree given by job seekers to jobs $\left(\bar{B}_{q}=\left[\bar{b}_{q i j}\right]_{n \times m}\right)$

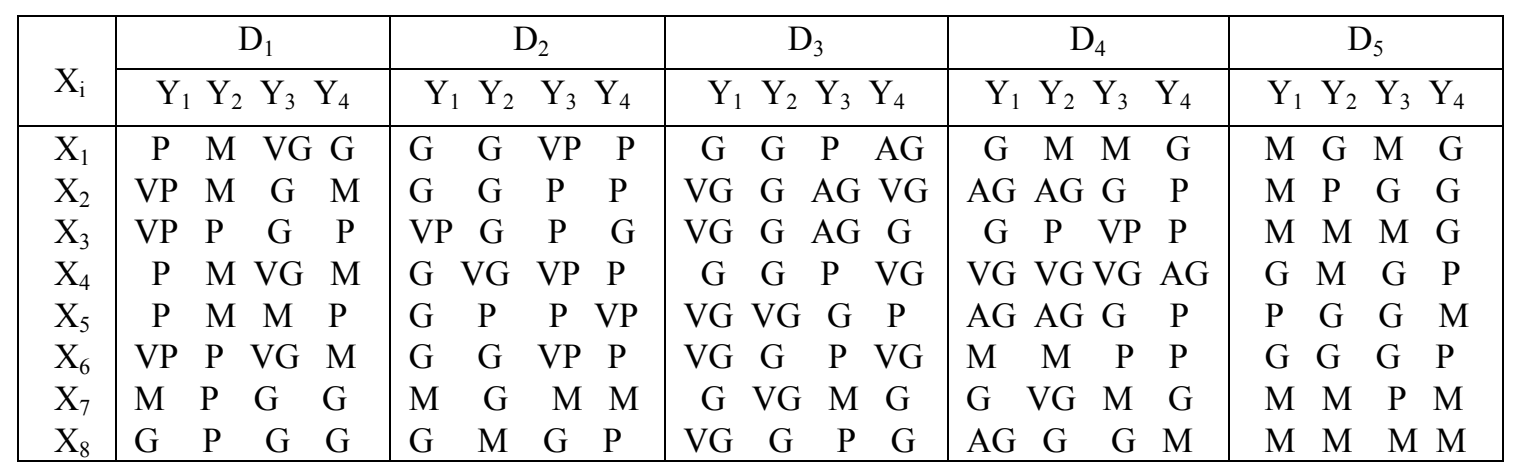


Table 4. Fuzzy comprehensive evaluation value of matching satisfaction degree given by jobs to job seekers $\tilde{\alpha}_{i j}=\left(\alpha_{i j}^{l}, \alpha_{i j}^{s}, \alpha_{i j}^{r}\right)$

\begin{tabular}{|l|l|l|l|c|}
\hline $\mathrm{X}_{\mathrm{i}}$ & $\mathrm{Y}_{1}$ & $\mathrm{Y}_{2}$ & $\mathrm{Y}_{3}$ & $\mathrm{Y}_{4}$ \\
\hline $\mathrm{X}_{1}$ & $(0.134,0.300,0.467)$ & $(0.134,0.300,0.467)$ & $(0.167,0.334,0.500)$ & $(0.100,0.267,0.433)$ \\
$\mathrm{X}_{2}$ & $(0.367,0.533,0.700)$ & $(0.367,0.533,0.700)$ & $(0.367,0.533,0.700)$ & $(0.400,0.567,0.734)$ \\
$\mathrm{X}_{3}$ & $(0.433,0.600,0.767)$ & $(0.600,0.767,0.900)$ & $(0.434,0.600,0.700)$ & $(0.467,0.634,0.800)$ \\
$\mathrm{X}_{4}$ & $(0.367,0.533,0.700)$ & $(0.300,0.467,0.633)$ & $(0.400,0.567,0.733)$ & $(0.267,0.433,0.600)$ \\
$\mathrm{X}_{5}$ & $(0.567,0.733,0.867)$ & $(0.633,0.800,0.933)$ & $(0.500,0.633,0.767)$ & $(0.533,0.700,0.833)$ \\
$\mathrm{X}_{6}$ & $(0.400,0.567,0.700)$ & $(0.367,0.534,0.667)$ & $(0.400,0.567,0.734)$ & $(0.300,0.467,0.633)$ \\
$\mathrm{X}_{7}$ & $(0.400,0.567,0.733)$ & $(0.400,0.567,0.733)$ & $(0.433,0.600,0.767)$ & $(0.400,0.567,0.733)$ \\
$\mathrm{X}_{8}$ & $(0.433,0.600,0.767)$ & $(0.433,0.600,0.767)$ & $(0.400,0.567,0.733)$ & $(0.267,0.433,0.600)$ \\
\hline
\end{tabular}

Table 5. Fuzzy comprehensive evaluation value of matching satisfaction degree given by job seekers to jobs $\tilde{\beta}_{i j}=\left(\beta_{i j}^{l}, \beta_{i j}^{s}, \beta_{i j}^{r}\right)$

\begin{tabular}{|l|l|l|l|c|}
\hline $\mathrm{X}_{\mathrm{i}}$ & $\mathrm{Y}_{1}$ & $\mathrm{Y}_{2}$ & $\mathrm{Y}_{3}$ & $\mathrm{Y}_{4}$ \\
\hline $\mathrm{X}_{1}$ & $(0.400,0.567,0.733)$ & $(0.433,0.600,0.767)$ & $(0.300,0.467,0.633)$ & $(0.500,0.667,0.800)$ \\
$\mathrm{X}_{2}$ & $(0.467,0.633,0.767)$ & $(0.467,0.633,0.767)$ & $(0.500,0.667,0.800)$ & $(0.367,0.533,0.700)$ \\
$\mathrm{X}_{3}$ & $(0.300,0.467,0.633)$ & $(0.333,0.500,0.667)$ & $(0.367,0.533,0.667)$ & $(0.367,0.533,0.700)$ \\
$\mathrm{X}_{4}$ & $(0.467,0.633,0.800)$ & $(0.500,0.667,0.833)$ & $(0.400,0.567,0.733)$ & $(0.433,0.600,0.733)$ \\
$\mathrm{X}_{5}$ & $(0.467,0.633,0.767)$ & $(0.500,0.667,0.800)$ & $(0.400,0.567,0.733)$ & $(0.167,0.333,0.500)$ \\
$\mathrm{X}_{6}$ & $(0.400,0.567,0.733)$ & $(0.400,0.567,0.733)$ & $(0.300,0.467,0.633)$ & $(0.300,0.467,0.633)$ \\
$\mathrm{X}_{7}$ & $(0.400,0.567,0.733)$ & $(0.467,0.633,0.800)$ & $(0.333,0.500,0.667)$ & $(0.433,0.600,0.767)$ \\
$\mathrm{X}_{8}$ & $(0.567,0.733,0.867)$ & $(0.367,0.533,0.700)$ & $(0.400,0.567,0.733)$ & $(0.367,0.533,0.700)$ \\
\hline
\end{tabular}

Table 6. Six positive and negative ideal solution of objective function

\begin{tabular}{|lcccccc|}
\hline & $z_{1}^{\prime}$ & $z_{1}^{\prime \prime}$ & $z_{1}^{\prime \prime \prime}$ & $z_{2}^{\prime}$ & $z_{2}^{\prime \prime}$ & $z_{2}^{\prime \prime \prime}$ \\
\hline positive ideal solution & 3.433 & 1.336 & 2.636 & 3.767 & 1.336 & 2.667 \\
negative ideal solution & 0 & 0 & 0 & 0 & 0 & 0 \\
\hline
\end{tabular}




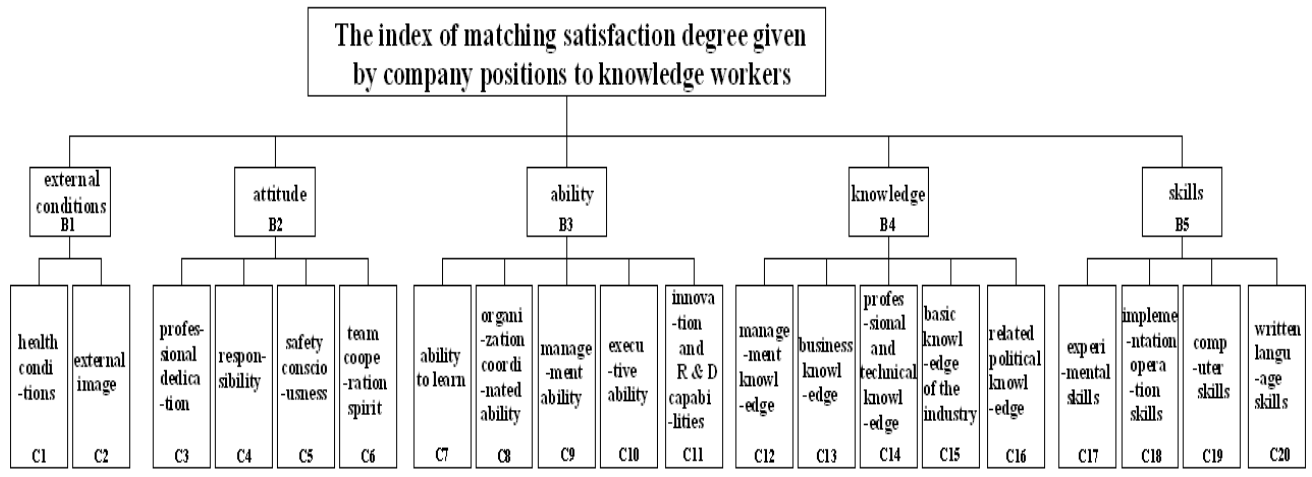

Figure 1. Competency model of job satisfaction

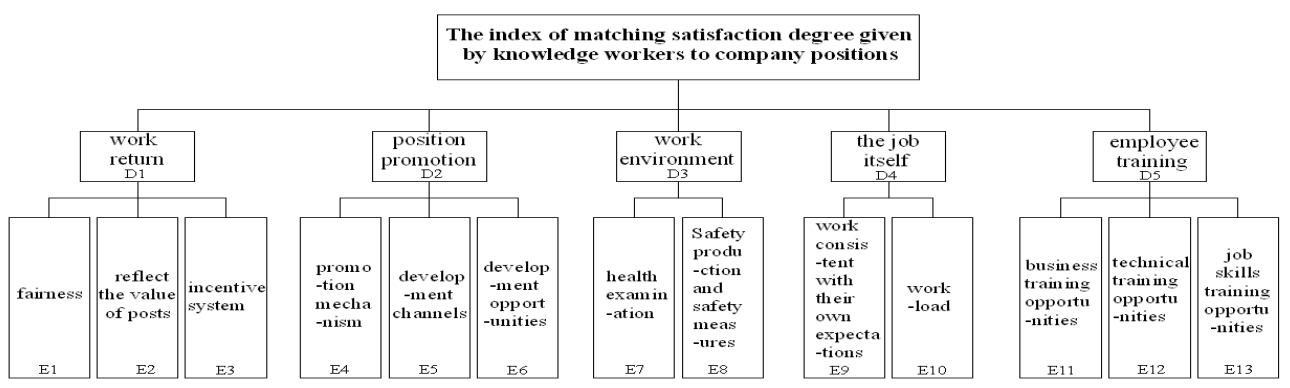

Figure 2. Job seekers satisfaction indicator system 